\title{
A pilot study on non-invasive treatment of migraine: The self- myofascial release
}

\author{
Alessandra Amato (1), Giuseppe Messina (1), Valerio Giustino (1), Jessica Brusa (1), \\ Filippo Brighina (2), Patrizia Proia (1)
}

(1) Department of Psychological, Pedagogical and Educational Sciences, Sport and Exercise Sciences Research Unit, University of Palermo, Palermo, Italy;(2) Department of Experimental Biomedicine and Clinical Neurosciences (BIONEC), University of Palermo, Palermo, Italy

This article is distributed under the terms of the Creative Commons Attribution Noncommercial License (CC BY-NC 4.0) which permits any noncommercial use, distribution, and reproduction in any medium, provided the original author(s) and source are credited.

\begin{abstract}
The aims of this paper was to determine the effect of self-myofascial release (SMFR) on postural stability and to analyze if it can influence migraine condition. Twenty-five subjects (age $49.7 \pm$ 12.5) affected by migraine were enrolled. Assessments included a stabilometric analysis in order to evaluate balance and plantar support, with eyes open (OE) and closed (CE); cervical ROM measurement; evaluation of upper limb strength through handgrip. All the analysis were carried out before and after the administration of a single SMFR protocol, using medium density small balls laid in the three most painful trigger points in migraine patients: trapezius, sternocleidomastoids and suboccipital muscles. Performing a T test for paired samples, there was a significant increase in two ranges of the stabilometric analysis: ellipse surface, both with open and closed eyes ( $\mathrm{p}$ value EO $=0.05$; $\mathrm{p}$ value EC $=0.04$ ) and length of the sway path, but just with closed eyes ( $p$ value $=0.05$ ). SMFR might have a positive impact on postural stability in subjects with migraine. Further investigation should be conducted to confirm the hypothesis.
\end{abstract}

Key Words: migraine; myofascial release techniques, fascia, balance, cervical ROM

Eur J Transl Myol 31 (1): 9646, 2021 doi: 10.4081/ejtm.2021.9646

The Myofascial Release (MFR) is a method that allows, autonomously, to treat connective tissue, relaxing contracted and painful muscles, on which depend on changes in strength and posture. A recent review from Kalichman and coll. ${ }^{1}$ shows that MFR improves the range of motion and the quality of movements, reduces tissue adhesions, decreases muscle hypertonus, helps tissues and prevents injuries. MFR seems to have therefore a series of potentially valuable effects, both for athletes and for the general population suffering from various diseases, such as migraine which often has accompanying symptoms like musculoskeletal impairments like cervical range of movement (ROM) limitations or impaired balance. But MFR is operatorsensitive technique and the subject who needs the intervention often cannot decide independently when and where to carry out the treatment. Instead, Selfmyofascial release (SMFR) is performed under the same principles of MFR but instead of a therapist providing soft-tissue manual therapy, an individual treats him/herself. The simpleness of SMFR allow it to be easily used in any place and at the time of need such as administering a drug therapy. It's shown that MFR can help in postural reorganization. ${ }^{2}$ In migraine the relaxation of suboccipital muscles through MFR and the consequent increase in cranial vascularization can help in symptoms management. ${ }^{3}$ We investigated posture, general upper limbs strength and ROM because cervical musculoskeletal impairments and balance disorders are often impaired function in headache sufferers. ${ }^{4,5}$

Migraine is a CNS disorder that involves some neurotransmitters and some pain circuits and is part of the larger class of "primary headaches", which indicate all types of pain at the head of a benign type, characterized by the lack of real pathology in other words it is a recognizable organic cause; migraine is a common disorder of neurovascular system, characterized by disabling and recurrent headache, commonly referred unilateral. Even if we do not know the exact mechanism of the headache, it is known that the triggering factors are numerous. Among the most frequent we find: female hormonal variations, stress, intense or flashing lights, smells and perfumes, loud noises and sounds but some foods and drinks and many others. Advances in understanding migraine genetic predisposition and the discovery of multiple susceptible genetic variants define 
the most convincing hypothesis of generalized neuronal hyperexcitability, given the anatomical alterations seen in the migraine brain. ${ }^{6}$ It is thought that MFR technique in general, can acts on tissues, mobilizing the hypodermis, modifying the consistency of the fundamental substance of the deep fascia, restoring the sliding between the endofascial collagen fibers, breaking the adhesions between the various layers of the deep bands, recreating the elasticity of the connective tissue skeleton. ${ }^{7}$ In fact, fibroblasts are able to reshape their cytoskeleton and in particular, if they're subjected to mechanical stress variations, they implement an actinic redistribution and an acto-myosin contraction in a few minutes. ${ }^{8,9}$ Myofibroblastic activity is highly sensitive to oxygen levels, vasoactive peptide, autonomic activity, pro-inflammatory cytokines and surrounding tissue tension. ${ }^{10}$ This could lead to an architectural alteration also in the surrounding tissue, increasing stress fibers (actin contractile fibers detectable in non-muscular cells) and coupling to focal adhesions (macromolecular assemblies through which mechanical forces and regulatory signals are transmitted between the extracellular matrix and an interacting cell). ${ }^{11}$ Therefore, any deregulation of the mechanisms by which cells get mechanical signals converting them into chemical response, can lead to dysregulation of cellular metabolic processes and degradation of connective tissue components. ${ }^{12}$ This can lead to the increased or decreased of the extracellular matrix deposition (ECM), altering tissue architecture and, in some cases, can inducing significant morbidity. ${ }^{13}$ Furthermore, it has been well know that in chronic musculoskeletal conditions, a change in the thickness of the deep fascial layer is related to an increase of loose connective tissue between the layers of dense collagen fibers, without an evident increase of the collagen itself. ${ }^{14}$ This can occur through the interaction between the autonomic fibers and smooth muscle cells located in the fascia that can contract as smooth muscle tissue. ${ }^{15}$ It has been suggested that sympathetic activation can induce myofibroblastic contraction in the fascial tissue by the release of TGF- $\beta 1$, as well as other cytokines can modulate the fascial stiffness. ${ }^{16}$ It's shown that MFR significantly increases joint flexibility and the range of movement (ROM) without causing any reduction in strength or decreases in performance. ${ }^{17}$ Simplicity and availability of small tools used for this type of treatment (small massaging balls, foam roller, medical balls, etc.) makes it easily and allow the subject treats him/herself. However, many papers have studied the effects of MFR but none have considered the effects of SMFR in subjects with migraines. Definitely, the aim of our study was to analyse the effects of a single SMFR protocol (Figure 1) on subjects with a diagnosis of migraine, through the posturometric and strength analysis to verify if treatment can be suggested to reduce musculoskeletal impairments, improve ROM cervical and balance, delay chronic therapy, and decrease drug intake in line with non-drug treatment studies for migraine. ${ }^{18}$

\section{Materials and Methods}

\section{Participants}

Twenty-five subjects (19 F, $6 \mathrm{M}$; age $49.7 \pm 12.5$, height $164.9 \pm 6.62 \mathrm{~cm}$, weight $68.6 \pm 16.9 \mathrm{~kg}$ ) were enrolled in our study. All subjects had positive diagnosis of migraine diagnosed by a neurologist through a specialist medical examination fulfilling diagnostic criteria for migraine without aura according to the international classification of Headache Disorder 3rd version (ICHD III). ${ }^{19}$ Subjects had an average number of attacks/months $6.48 \pm 2.02$ with 16,32 \pm 7,50 years of migraine diagnosis. Exclusion criteria were: pregnancy, other major diseases like diabetes, rheumatic and genetic diseases.

\section{Design}

This is a quasi-experimental study aiming to establish the effectiveness of health-related interventions. The protocol involved the subjects that were not under therapy, but were in the diagnostic phase. The compilation of the medical history (questionnaire of the most painful trigger point) was performed under the neurologist supervsion; the analysis with a stabilometric platform (Sensor Medica ${ }^{\circledR}$ Maxi 40x40 Software by Freestep), cervical ROM assessment through an accelerometer (Moover ${ }^{\circledR}$; Sensor Medica ${ }^{\circledR}$; Guidonia Montecelio, Roma, Italia) and evaluation of upper limb strength through handgrip test (KERN MAP Version 1.2 08/2012, Hand Grip Dynamometer) were repeated before and after the SMFR treatment.

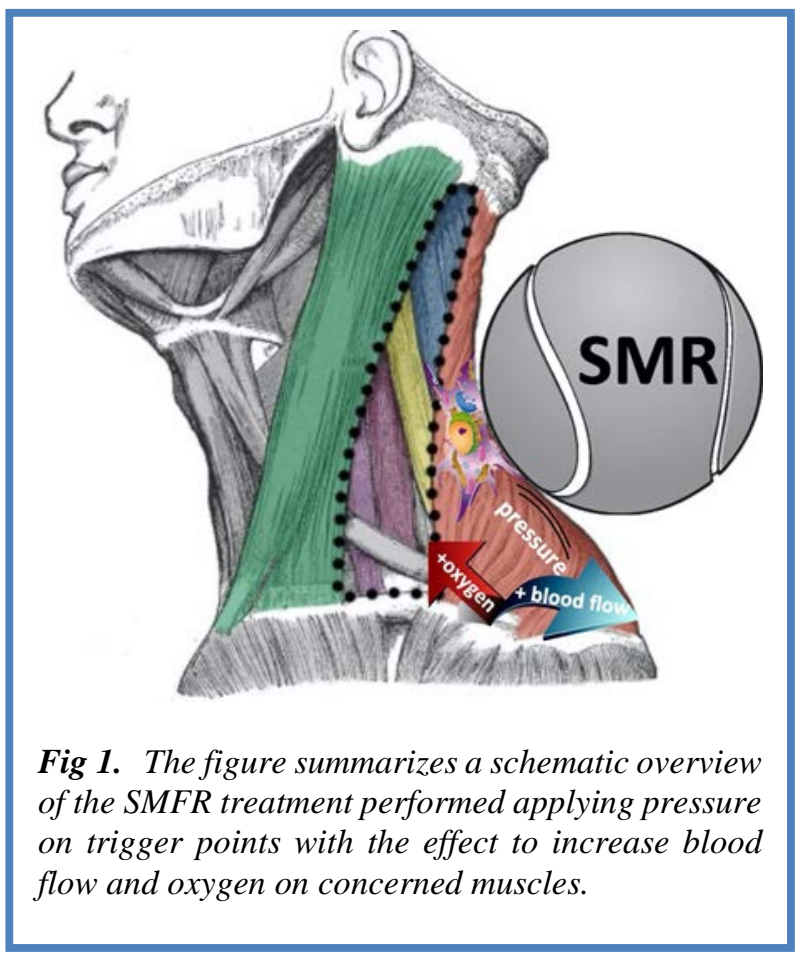




\section{Assessments}

In postural analysis, the subject is placed in an orthostatic position with the arms along the sides, on the platform with feet parallel for 5 seconds. After that the stabilometric evaluation assessed the oscillations of the body and consists in placing the subject on platform in an orthostatic position with the arms at the sides with the heels about $4 \mathrm{~cm}$ apart one from the other and with the forefoot pointing outward with an angle of $30^{\circ}$ for 51.1 seconds, in accordance with the Romberg's test. This assessment was done first with open (OE) and then closed eyes (CE), to see how the values changed by inhibiting the eye, very important to maintain posture.

To test the cervical ROM, each participant, seated in a chair, performed neck movements on the three planes until the maximal ROM measured via a non-invasive technique using a wireless computer-aided accelerometer positioned medially of the frontal bone of the skull and above the bridge of the nose and fastened around the head through a strap.

The handgrip test was done with the patient sitting in neutral position, with his feet parallel well placed on the ground and with the arm bent at $90^{\circ}$ without any kind of support. The subject had to press maximally the electronic spring dynamometer for three seconds, alternating right hand and left hand. Between each series a minute of recovery was taken. The resistance of the spring of the handgrip was changed according to the subject: $80 \mathrm{~kg}$ for men and $40 \mathrm{~kg}$ for women.

\section{Self-Myofascial Release protocol}

The SMFR consisted of a static myofascial release, carried out with the subject supine with knees flexed using special medium-density sponge balls, laid with the only gravity pressure, for 90 seconds in precise body areas: trapezius, sternocleidomastoids and sub-occipital muscle groups. ${ }^{20,21}$ This body areas were selected for SMFR treatment due to the high frequency correlations between headache pain and refer pain in these Myofascial trigger points, in these muscles, during migrain attack. ${ }^{22-}$ ${ }^{25}$ Before the start of the protocol a physical exercise expert showed to the subjects the exact points where to place the ball, then they did by themselves (Figure 1). The participants moved ball proximally to distally in small undulating movements (approximately one per second) for 30 seconds, ${ }^{26}$ followed by 10 -seconds rest between compressions to allow blood reperfusion. This maneuver was repeated for 3 sessions, ${ }^{25} 30$ seconds each, for a total of 90 seconds for each Myofascial trigger points performed in the muscles considered and for each side (left and right divided by the spine). Therefore, the SMFR treatment lasted 9 minutes for subject.

\section{Statistical Analysis}

Differences between the assessments results before and after treatment were analyzed by student's t-test for paired sample. Calculations and tables were all made in SPSS (IBM SPSS Statistics 23). Data are shown as means and SEM. Differences resulting in a $\mathrm{P}$ value $<0.05$ were considered statistically significant.

\section{Ethical considerations}

The study was approved by ethics committee Palermo 1, Policlinico Giaccone Hospital, Palermo, Italy, (ethic code: PA01; 12/2014) and was conducted in accordance with the declaration of Helsinki. Informed written consent was obtained from all subjects involved in the study. A medical history questionnaire was collected during the time in wich the subjects underwent medical examination.

\section{Results and Discussion}

The descriptive statistical analysis of our samples showed the load distribution (\%) measured by the baropodometry of $52.5 \pm 4.09$ in the right foot and 47.5 \pm 4.09 in the left foot. As regard the different distribution between the forefoot and rearfoot was respectively: 55.7 $\pm 5.94 \%$ and $44.3 \pm 5.95 \%$ for the right foot and $52.7 \pm$ $5.12 \%$ and $47.3 \pm 5.12 \%$ for the left foot. Performing a $T$ test for paired samples we highlighted a significant increase in two ranges of the stabilometric analysis (Tab.1): ellipse surface, expressed in $\mathrm{mm}^{2}$, both with open and closed eyes (pre 172,63 \pm 287,25; post 64,31 \pm 43,63 ; $p$ value $=0.05(\mathrm{OE})$; pre 166,1 \pm 349; post 111,3 $\pm 251,1 ; p$ value $=0.04$, (CE)) and length of the sway path, but only with closed eyes (pre 465,3 $\pm 165,6$; post $431,86 \pm 109,5 ; p$ value $=0.05$ ).

Concerning the results of the cervical ROM and handgrip test we didn't find any significant changes after the SMFR treatment (Table 1).

The aim of the study was to evaluate the postural and motor parameters of subjects who suffer of migraine in order to understand whether they have postural and functional characteristics equivalent to a healthy population and if an SMFR protocol can influence their balance, cervical ROM and strength. The analysis of the data showed that the evaluated subjects have a load distribution, compared with the reference values, quite normal. As far as the forefoot- rearfoot distribution is concerned they are presented with anteriorized respect to the reference values as follows: $46 \%$ in the rearfoot and $54 \%$ in the forefoot. ${ }^{27}$ The improvement in stabilometry with the decrease of Ellipse surface $\mathrm{OE}$ and $\mathrm{CE}(\mathrm{p}<0,05)$ and of length of the sway path $(\mathrm{CE})(\mathrm{p}<0,05)$ could be due to the release through the SMFR of the neck muscles, which are certainly more involved in this type of pathology and that contribute in maintaining balance. Therefore, this could support the hypothesis that SMFR could become an important technique to manage altered balance that is one of the accompanying symptoms. ${ }^{28}$ Values detected for cervical ROM showed a lightly trend 
Table 1. Mean and standard deviation of pre and post SMFR treatment of stabilometry parameters (open eyes (OE) and closed eyes (CE)), ROM parameters and handgrip test results.

\begin{tabular}{llll}
\hline & \multicolumn{1}{c}{ Pre } & \multicolumn{1}{c}{ Post } & P value \\
\hline Ellipse surface (OE) $\mathbf{m m}^{2}$ & $172,63 \pm 287,25$ & $64,31 \pm 43,63$ & $0,05^{*}$ \\
\hline Length of the sway path (OE) $\mathbf{m m}$ & $411,19 \pm 143,73$ & $378,23 \pm 63,30$ & 0,17 \\
\hline Ellipse surface (CE) $\mathbf{m m}^{2}$ & $166,1 \pm 349$ & $111,3 \pm 251,1$ & $0,0 *^{*}$ \\
\hline length of the sway path (CE) mm & $465,3 \pm 165,6$ & $431,86 \pm 109,5$ & $0,05^{*}$ \\
\hline Right Cervical ROM Rotation (degrees) & $59,7 \pm 13,4$ & $57,06 \pm 12,5$ & 0,22 \\
\hline Left Cervical ROM Rotation (degrees) & $63,8 \pm 15,5$ & $66,9 \pm 14$ & 0,09 \\
\hline Right Cervical ROM Side bending (degrees) & $32,8 \pm 10,5$ & $32,2 \pm 10,1$ & 0,64 \\
\hline Left Cervical ROM Side bending (degrees) & $35,9 \pm 8,6$ & $35,9 \pm 11$ & 0,98 \\
\hline Cervical ROM flexion ( degrees) & $42,9 \pm 11,5$ & $42,1 \pm 9,9$ & 0,60 \\
\hline Cervical ROM extention (degrees) & $56,3 \pm 14$ & $57,5 \pm 13,6$ & 0,48 \\
\hline Right Handgrip test (Kg) & $22,8 \pm 10,5$ & $22,2 \pm 10,8$ & 0,31 \\
\hline Left Handgrip test (Kg) & $22 \pm 9,1$ & $21,03 \pm 8,5$ & 0,36 \\
\hline
\end{tabular}

* Significant differences at the 0.05 level

in increase for the left rotation $(\mathrm{p}=0.09)$. It is known that migraine is associated with muscle pain in the neck region and patient who suffered of this disorder adopt an antalgic posture modifying the cranio-cervical posture. ${ }^{7}$ This condition causes asymmetrical muscular tensions. A possible explanation for the improvement on cervical rotation in patients with migraine may be explained because the SMFR treatment technique decreased muscle stiffness caused by the irritation of head and neck sensory nerves. This treatment might have a positive impact on cervical mobility and involves an improvement on physical fitness in people who suffer of this disorder. ${ }^{29}$ Maybe to a have a statistical significant variation it is necessary to treat the patients for more than one time. Moreover during the treatment the patients were not in an acute headache phase and this might affect the result. Further investigation should be conducted to confirm the hypothesis. The analysis in the maximum grip test with handgrip showed a lower strength than the standard strength found in literature for both hands. An article, published in 2013, ${ }^{30}$ examined subjects aged between 45 and 60 years and the standard levels of handgrip of left hand had an average of 37.16; while the average standard levels of the right hand were 40 while in our sample was in mean 22, but no changes are shown between pre and post SMFR protocol treatment in the handgrip test ( $>0.05$ ); this supports the assertion by Connolly et al. who affirm that a single protocol of MFR technique (with Foam Rolling in this case) increases ROM without compromising strength. ${ }^{26}$ The decrease in strength and a lower capacity for rotation and side bending of the cervical area compared to the normal range demonstrates the involvement of these structures in the pathologies. ${ }^{30}$ It is in fact known that pain, especially in the musculoskeletal system, involves muscular retractions located in the algic site and not an antalgic strategy. A retracted muscle appears to be in a basal muscleshortening situation with a consequent decrease in its flexibility leading to a limitation in joint excursion and in the mobility: of course the final consequence is an imbalance between the agonist and antagonist muscles. This imbalance involves an almost continuous stiff state of muscles inducing high muscle tension. In conclusion to confirm the preliminary results probably it would be necessary to try performing the SMFR treatment more than once, perhaps in a more acute phase of pain that allow better identification of the trigger points responsible for the pain.

This study has some limitations that should be acknowledged. The quasi-experimental design it would need a control group whilst here we had just one group. Postural assessments were made only twice in the pre and post period and maybe it would be necessary repeat more than two time these measurements to verify the 
maintenance of the effects of SMFR over time. It should also be taken into account that these results refer to only this target population. Investigation are needed to clarify if these effects are sex and ages correlated and to confirm them increasing the number of subjects investigated.

\section{List of acronyms}

SMFR - self-myofascial release

$\mathrm{OE}$ - open eyes

CE - closed eyes

ROM - range of movement

MFR - Myofascial Release

CNS - central nervous system;

ECM - extracellular matrix deposition;

TGF- $\beta 1$ - Transforming Growth Factor $\beta 1$

ICHD - International Classification of Headache Disorder

\section{Authors contributions}

GM, AA and PP contributed to study design and wrote the manuscript. VG, JB and FB participated in data collection and analysis. JB, VG and AA performed analysis and interpretation of results. GM and PP reviewed literature and contributed to the interpretation of data. All authors critically reviewed final and draft approved the submitted typescript.

\section{Acknowledgments}

The authors wish to thanks all the subjects that participated to the study and all the neurologists that helped us to recruit the patients. However, we would also to thanks the students that participated and supported to collect the data in particular Alessandra Lo Monaco e Enrico Costanzo.

\section{Funding None}

\section{Conflict of Interest}

The authors declare no competing interests.

\section{Ethical Publication Statement}

We confirm that we have read the Journal's position on issues involved in ethical publication and affirm that this report is consistent with those guidelines.

\section{Corresponding Author}

Patrizia Proia, Department of Psychological, Pedagogical and Educational Sciences, Sport and Exercise Sciences Research Unit, University of Palermo, I-90128, Italy ORCID iD: 0000-0002-0326-5560

E-mail: patrizia.proia@unipa.it

\section{E-mail and ORCID of co-authos}

Alessandra Amato: alessandra.amato02@unipa.it ORCID iD: 0000-0002-6512-3840

Giuseppe Messina: giuseppe.messina17@unipa.it ORCID iD: 0000-0003-2774-4950

Valerio Giustino: valerio.giustino@unipa.it ORCID iD: 0000-0002-4575-8021
Jessica Brusa: brusajessica@gmail.com ORCID iD: none

Filippo Brighina: filippo.brighina@unipa.it ORCID iD: 0000-0001-5875-3126

\section{References}

1. Kalichman L, Ben David C. Effect of SelfMyofascial Release on Myofascial Pain, Muscle Flexibility, and Strength: A Narrative Review. 2017 Apr;21(2):446-451. doi: 10.1016/j.jbmt.2016.11. 006. Epub 2016 Nov 14.

2. Rutherford G, Manning P, Newton JL. Understanding Muscle Dysfunction in Chronic Fatigue Syndrome. J Aging Res. 2016;2016: 2497348. doi: 10.1155/2016/2497348. Epub 2016 Feb 22.

3. Burstein R, Noseda R, Borsook D. Migraine: Multiple Processes, Complex Pathophysiology. J Neurosci. 2015 Apr 29;35(17):6619-29. doi: 10. 1523/JNEUROSCI.0373-15.2015.

4. $\quad$ Liang Z, Galea, O, Thomas L, Jull G, Treleaven J. Cervical Musculoskeletal Impairments in Migraine and Tension Type Headache: A Systematic Review and Meta-Analysis. Musculoskelet Sci Pract. 2019 Jul;42:67-83. doi: 10.1016/j.msksp.2019.04.007. Epub 2019 Apr 25.

5. Baraldi C, Gherpelli C, Alicandri Ciufelli M, Monzani D, Pini LA, Pani L.,Guerzoni S. A CaseControl Study of Visually Evoked Postural Responses in Childhood with Primary Headaches. Neurol Sci. 2020 Feb;41(2):305-311. doi: 10. 1007/s10072-019-04072-2. Epub 2019 Sep 9.

6. Blaschek A, Milde-Busch A, Straube A, chankin C, Langhagen T, Jahn K, Schröder SA, Reiter K, von Kries R, Heinen F. Self-Reported Muscle Pain in Adolescents with Migraine and Tension-Type Headache. Cephalalgia. 2012 Feb;32(3):241-9. doi: 10.1177/0333102411434808. Epub 2012 Jan 16.

7. Beardsley C, Škarabot J. Effects of Self-Myofascial Release: A Systematic Review. J Bodyw Mov Ther. 2015 Oct;19(4):747-58. doi: 10.1016/j.jbmt.2015. 08.007. Epub 2015 Aug 28.

8. Langevin HM, Sherma, KJ. Pathophysiological Model for Chronic Low Back Pain Integrating Connective Tissue and Nervous System Mechanisms Med Hypotheses. 2007;68(1):74-80. doi: 10.1016/j.mehy.2006.06.033. Epub 2006 Aug 21

9. Fernández-de-las-Peñas C, Cuadrado M, Pareja J. Myofascial Trigger Points, Neck Mobility and Forward Head Posture in Unilateral Migraine. Cephalalgia. 2006 Sep;26(9):1061-70. doi: 10.1111 /j.1468-2982.2006.01162.x.

10. Langevin HM, Storch KN, Cipolla MJ, White SL, Buttolph TR, Taatjes DJ. Fibroblast Spreading Induced by Connective Tissue Stretch Involves Intracellular Redistribution of $\alpha$ - and $\beta$-Actin. Histochem Cell Biol. 2006, May;125(5):487-95. doi: 10.1007/s00418-005-0138-1. Epub 2006 Jan 14. 
11. Kreis TE, Birchmeier W. Stress Fiber Sarcomeres of Fibroblasts Are Contractile. Cell. 1980 Nov;22(2 Pt 2):555-61. doi: 10.1016/0092-8674(80)90365-7.

12. Ingber D. Mechanobiology and Diseases of Mechanotransduction. Ann Med. 2003;35(8):56477. doi: 10.1080/07853890310016333.

13. McAnulty RJ. Fibroblasts and Myofibroblasts: Their Source, Function and Role in Disease. Int J Biochem Cell Biol. 2007;39(4):666-71. doi: 10. 1016/j.biocel.2006.11.005.

14. Stecco A, Meneghini A, Stern R.; Stecco C, Imamura M. Ultrasonography in Myofascial Neck Pain: Randomized Clinical Trial for Diagnosis and Follow-Up. Surg Radiol Anat. 2014 Apr;36(3):24353. doi: 10.1007/s00276-013-1185-2. Epub 2013 Aug 23.

15. Schleip R, Klingler W, Lehmann-Horn F. Active Fascial Contractility: Fascia May Be Able to Contract in a Smooth Muscle-like Manner and Thereby Influence Musculoskeletal Dynamics. Med Hypotheses. 2005;65(2):273-7. doi: 10.1016/j. mehy.2005.03.005.

16. Porter KE, Turner NA. Cardiac Fibroblasts: At the Heart of Myocardial Remodeling. Pharmacol Ther. 2009 Aug;123(2):255-78. doi: 10.1016/j.pharm thera.2009.05.002. Epub 2009 May 19.

17. Schleip R, Duerselen L, Vleeming A, Naylor IL, Lehmann-Horn F, Zorn A, Jaeger H, Klingler W. Strain Hardening of Fascia: Static Stretching of Dense Fibrous Connective Tissues Can Induce a Temporary Stiffness Increase Accompanied by Enhanced Matrix Hydration. J Bodyw Mov Ther. 2012, Jan;16(1):94-100. doi: 10.1016/j.jbmt.2011. 09.003. Epub 2011 Dec 5.

18. Fernández-de-las-Peñas C, Florencio LL, PlazaManzano G, Arias-Buría JL. Clinical Reasoning Behind Non-Pharmacological Interventions for the Management of Headaches: A Narrative Literature Review. Int J Environ Res Public Health. 2020 Jun 9;17(11):4126. doi: 10.3390/ijerph17114126.

19. Headache Classification Committee of the International Headache Society (IHS) The International Classification of Headache Disorders, 3rd Edition (Beta Version). Cephalalgia. Jul;33(9):629-808. doi:10.1177/0333102413485658.

20. Moraska A, Chandler C. Changes in Clinical Parameters in Patients with Tension-Type Headache Following Massage Therapy: A Pilot Study. J Man Manip Ther. 2008;16(2):106-12. doi: 10.1179/106698108790818468.

21. Hou C.-R, Tsai L-C, Cheng K-F, Chung K-C, Hong C-Z. Immediate Effects of Various Physical Therapeutic Modalities on Cervical Myofascial Pain and Trigger-Point Sensitivity. Arch Phys Med Rehabil. 2002 Oct;83(10):1406-14. doi: 10.1053 /apmr.2002.34834.

22. Fernandez-de-las-Penas C, Alonso-Blanco C, Cuadrado ML, Gerwin RD, Pareja JA. Myofascial
Trigger Points and Their Relationship to Headache Clinical Parameters in Chronic Tension-Type Headache. 2006 Sep;46(8):1264-72. doi: 10.1111 /j.1526-4610.2006.00440.x.

23. Alonso-Blanco C, de-la-Llave-Rincón AI, Fernández-de-las-Peñas C. Muscle Trigger Point Therapy in Tension-Type Headache. Expert Rev Neurother. 2012 Mar;12(3):315-22. doi: 10.1586/ ern.11.13.

24. Fernández-de-las-Peñas, C.; Ge, H.-Y.; AlonsoBlanco, C.; González-Iglesias, J.; Arendt-Nielsen, L. Referred Pain Areas of Active Myofascial Trigger Points in Head, Neck, and Shoulder Muscles, in Chronic Tension Type Headache. J Bodyw Mov Ther. 2010, Oct;14(4):391-6. doi: 10.1016/j.jbmt.2009.06.008. Epub 2009 Aug 3.

25. Moraska AF, Stenerson L, Butryn N, Krutsch JP, Schmiege SJ, Mann JD. Myofascial Trigger PointFocused Head and Neck Massage for Recurrent Tension-Type Headache: A Randomized, PlaceboControlled Clinical Trial. Clin J Pain. Feb; 31(2):159-68. doi: 10.1097/AJP.0000000000 000091.

26. Connolly G, Hammer RL, Powell JA, O’Connor PL. A Single Bout of Foam Rolling Increases Flexibility of the Hip Adductor Muscles without Compromising Strength. Int J Exerc Sci. 2020 Aug 1;13(7):938-949. eCollection 2020.

27. Patti A, Bianco A, Şahin N, Sekulic D, Paoli A, Iovane A, Messina G, Gagey PM, Palma A. Postural Control and Balance in a Cohort of Healthy People Living in Europe: An Observational Study. Medicine (Baltimore). 2018 Dec;97(52):e13835. doi: 10.1097/MD.0000000000013835.

28. Carvalho GF, Schwarz A, Szikszay TM, Adamczyk WM, Bevilaqua-Grossi D, Luedtke K. Physical Therapy and Migraine: Musculoskeletal and Balance Dysfunctions and Their Relevance for Clinical Practice. Braz J Phys Ther. Jul-Aug 2020;24(4):306-317. doi: 10.1016/j.bjpt.2019.11.0 01. Epub 2019 Nov 29.

29. Messina G, Giustino V, Martines F, Rizzo S, Pirino, A, Scoppa F. Orofacial Muscles Activity in Children with Swallowing Dysfunction and Removable Functional Appliances. Eur J Transl Myol. 2019, Aug 27;29(3):8267. doi: 10.4081/ ejtm.2019.8267. eCollection 2019 Aug 2.

30. Wu H, Liu M, Zhang Q, Liu L, Meng G, Bao X, Gu Y, Zhang S, Sun S, Wang X, Zhou M, Jia Q, Song $\mathrm{K}$, Niu K Reference values for handgrip strength: data from the Tianjin Chronic Low-Grade Systemic Inflammation and Health (TCLSIH) cohort study. Age Ageing. 2020 Feb 27;49(2):233-238. doi: 10.1093/ageing/afz148.

Submission: January 28, 2020

Revision received: February 2, 2021 Accepted for publication: February 3, 2021 